Rapid Reviews COVID-19

\title{
Reviews of "Antisense \\ oligonucleotides target a \\ nearly invariant structural \\ element from the SARS- \\ CoV-2 genome and drive \\ RNA degradation"
}

Hasan Cubuk ${ }^{1}$, Anders Näär ${ }^{2}$, Valerio Orlando ${ }^{3}$

${ }^{1}$ Istanbul Arel University: Istanbul Arel Universitesi, Molecular Biology and Genetics, Turkey,

2University of California, Berkeley, Nutritional Sciences \& Toxicology, USA,

${ }^{3}$ KAUST, BESE, Saudi Arabia

Published on: Nov 05, 2020

DOI: $10.1162 / 2 \mathrm{e} 3983 f 5.9 f 85 a a 16$

License: Creative Commons Attribution 4.0 International License (CC-BY 4.0). 
To read the original manuscript, click the link above.

Summary of Reviews: This manuscript offers a gapmer-based therapeutic strategy targeting the highly conserved s2m element present in the SARS-CoV-2 genome. While potentially informative, the study's claims require testing with wildtype SARS-CoV-2 virus and animal models to be substantiated.

Reviewer 1 (Hasan Cubuk) | प्र०ण

Reviewer 2 (Anders Näär) | प्र० $\square \square$

Reviewer 3 (Valerio Orlando)

\author{
RR:C19 Strength of Evidence Scale Key \\ प्रमप्र = Misleading \\ प्र $\square \square \square=$ Not Informative \\ ㄷํ $\square \square=$ Potentially Informative \\ प्राप्र = Reliable \\ $\operatorname{l|c|c|c|}=$ Strong
}

To read the reviews, click the links below. 